\title{
LA REVISTA FEMINAL: PARADIGMA DE LAS PUBLICACIONES FEMINISTAS ESPAÑOLAS DE PRINCIPIOS DEL SIGLO XX
}

\author{
Feminal Magazine: Paradigm of Spanish Feminist Publications of the Early \\ Twentieth Century
}

\author{
Ana MuÑoz \\ Universidad de Salamanca \\ amufer@hotmail.com
}

Fecha de recepción: 23-II-2012

Fecha de aceptación: $10-I V-2012$

\begin{abstract}
Resumen: La revista Feminal se publicó como suplemento del periódico La Ilustració Catalana desde abril de 1907 hasta diciembre de 1917. La importancia de esta publicación estriba en ser la primera, en Barcelona, que trata cuestiones de relevancia para la historia del feminismo español como la reivindicación del derecho al voto. En la globalidad de la revista se aprecia, de forma explícita, el interés de su directora por aumentar la cultura de la mujer y el repertorio musical no es ajeno a este objetivo: en él se pueden apreciar diversas intenciones de Carmen Karr como el fomento de la autoría femenina, la creación de un vínculo entre autoras e intérpretes femeninas y la plasmación en la música de un determinado pensamiento cultural. El repertorio musical de la revista consigue crear una relación vinculante compartida por los participantes de la performance, configurándose una determinada identidad social femenina.
\end{abstract}

Palabras clave: feminismo, género, cancó catalana, mujer, revista Feminal, Cataluña.

AвsTRACT: The Feminal magazine was published as a supplement of the newspaper La Ilustració Catalana from April 1907 to December 1917. The mainstream of this publication lies in being the first, in Barcelona, which addresses issues of relevance to the history of Spanish feminism as the claiming for the voting rights. The magazine as the whole shows, explicitly, the interest of Karr to increase women's culture, in fact musical repertoire is no stranger to this goal: it can be seen several intentions of Carmen Karr such as the 
promotion of female authorship, creating a link between female authors and female interpreters and the translation in music of a particular cultural thought. The musical repertoire of the magazine creates a relationship shared by the members of the performance, building a particular female social identity.

Keywords: feminism, gender, Cataluña's cançó, women, Feminal magazine, Cataluña.

\section{INTRODUCCIÓN}

La revista Feminal surgió en un momento y lugar geográfico donde era necesaria una publicación que fuera reflejo del pensamiento cultural de una época pero con un punto de vista diferente, con el marginado hasta entonces de la cultura, con el punto de vista femenino.

La creación de Feminal se debió al proyecto personal de Carme Karr quien aunó en un mismo medio la reivindicación de la cultura catalana y la de los derechos de la mujer; surgiendo en 1907, en Barcelona, como suplemento del periódico la $\mathrm{I} /$-lustració Catalana y permaneciendo en las tiradas de imprenta durante diez años, momento en que desapareció debido a la crisis producida por la Primera Guerra Mundial.

Como la propia Karr enuncia en el primer número de la revista, su objetivo es producir una revista «destinada especialmente a la cultura de la mujer» ${ }^{1}$. Por ello, cualquier manifestación social, literaria, científica y artística realizada por una mujer tenía cabida en esta publicación mensual y en el terreno musical no sólo se comentaba la carrera profesional de diversas instrumentistas, cantantes y compositoras, sino que también se incluía una pieza musical en muchos de sus números ${ }^{2}$.

El repertorio musical publicado en Feminal pertenece al género de salón y es heredero de un conjunto de cambios que se dieron en las décadas precedentes en Cataluña, ya que la situación en el resto de España era

1 Ref. Carme Karr: Feminal (Barcelona: Establiment gràfich Thomas, 1907), 1, p. 2v. (Ana Muñoz, Trad.). A continuación transcribimos la cita original: «revista destinada especialment a la cultura de la dòna».

2 Para un mayor conocimiento sobre la revista Feminal, cfr. Isabel Segura: Els feminismes de "Feminal" (Barcelona: Institut Català de les Dones, 2007); Josep María Ainaud de Lasarte: Carme Karr (Barcelona: Infiesta, 2010) y la propia revista, Carme Karr: Op. Cit. 
diferente. El ascenso de la burguesía barcelonesa en el último tercio del siglo XIX, produjo una demanda de música pra este género y el reformismo político provocó que el arte catalán tuviera una mayor influencia europea. Asimismo, el aumento del interés por la recuperación de la cultura catalana, el florecimiento de la poesía catalana y el ideario de Felipe Pedrell sobre la canción lírica que conjugó sabia popular, romanticismo y europeísmo, ayudaron a los compositores catalanes a tomar consciencia de la trascendencia de la cançó catalana ${ }^{3}$ en el proceso de identificación nacional ${ }^{4}$.

En el primer apartado ofreceremos unas breves pinceladas de la revista Feminal, contextualizando el nacimiento de la revista y explicando su origen y las secciones en que se estructura para profundizar más tarde en el pensamiento ideológico de ella y poder comprender por qué la participación de la mujer fue tan importante para Feminal en cualquier campo relacionado con la cultura. Como parte de esta cultura, se encuentra la sección musical en la revista y debido a su relevancia como corpus musical en los salones barceloneses de principios del siglo XX consideramos apropiado su tratamiento en el último de los apartados del presente artículo.

\section{LA REVISTA FEMINAL: CONTEXTUALIZACIÓN, ORIGEN Y ESTRUCTURA}

La revista Feminal apareció en un momento donde tuvieron lugar una serie de cambios sociales y culturales debidos, en parte, a la industrialización. Tuvieron lugar varios acontecimientos, entre los que se encuentran el conflicto de clases, el de sexos y el renacimiento de la cultura catalana: el ascenso final de la burguesía catalana se produjo, en Barcelona, desde el último tercio del siglo XIX ${ }^{5}$; las reivindicaciones feministas, que comenzaron desde la segunda mitad del siglo XIX, tuvieron a partir del siglo XX una mayor relevancia ayudadas por influencias extranjeras y sobre la preocupación de

3 Esta denominación ha sido dada por Celsa Alonso. Cfr. Celsa Alonso: La canción lírica española en el siglo XIX (Madrid: ICCMU, 1988), p. 480.

4 Sobre el género de salón en el siglo XIX y la influencia de Felipe Pedrell en la configuración de la canco catalana véase el manual de Celsa Alonso (1988). Op. Cit., pp. $433-$ 485 y el artículo de Celsa Alonso: Los salones: un espacio musical para la España del XIX. Anuario musical: Revista de musicología del CSIC, 48, p. 167.

5 Ref. Celsa Alonso: Op. Cit, p. 480. 
la cultura catalana se apreció desde finales del siglo XIX un mayor auge vitalizado gracias a Valentí Almirall en Lo catalanisme $e^{6}$.

Feminal fue el proyecto personal de Carme Karr i Alfonsetti, escritora y colaboradora de varios periódicos como Joventut y L'Avenç, interés que compartía con su tío Alphonse Karr, prestigioso director del diario parisino Le Figaro. Lluís Via i Pagès, director literario de Joventut y fiel admirador de Karr, fue la persona que la vinculaba a las dos publicaciones y también fue quien le presentó a Francesc Matheu, director del periódico Il·lustració Catalana. Éste último fue quien recomendó a Karr que en lugar de crear una revista sólo para mujeres, utilizase un periódico ya establecido e hiciera un suplemento en él, consejo que siguió Carme Karr y a través de su periódico creó la revista Feminal'; publicación mensual que comenzó su existencia en 1907 y finalizó en 1917 debido al encarecimiento del papel por la segunda guerra mundial.

Carme Karr aunó dos de sus grandes de deseos en la revista: la reivindicación de la cultura catalana y la de los derechos de las mujeres. Sobre el primero de los deseos se interesó por cualquier manifestación cultural que se centrase en la región catalana, incluida la lengua, motivo por el cual toda la revista está escrita en catalán. Sobre el segundo de los deseos, a lo largo de la publicación se aprecia una gran participación del sector femenino en cualquier campo relacionado con la cultura; escribiendo Karr lo siguiente:

Que vengan (...) a nosotras todas las mujeres: las escritoras, las poetisas, pedagogas; las pintoras, dibujantes, músicas, las artistas, las sociólogas, las pacifistas, las estudiantes. Que vengan también las artesanas; que vengan sin timidez, todas aquellas que aspiren a ser algo. $O$ a producir cualquier obra social, artística, literaria, industrial, científica, etc., y traigan su pequeño grano de arena. ${ }^{8}$

6 Ref. Valentí Almirall: Lo catalanisme. Motius que'l llegitiman. Fonaments cientifichs y solucions prácticas (Barcelona: Alta Fulla, 1978).

7 Ref. Josep Maria Ainaud de Lasarte: Carme Karr (Barcelona: Infiesta, 2010), pp.17-27 y Ainaud de Lasarte, Josep María: "Carme Karr, escriptora i feminista", Serra d’Or, 409 (1984), pp. 20-23

8 Ref. Carme Karr: Feminal. Barcelona: Establiment gràfich Thomas, 45, p. 1v. (Ana Muñoz, trad.). A continuación, se transcribe la cita original: «la dona de Catalunya pot ventarse de tenir en la seva publicació femenina d'Espanya y de l'Amèrica llatina que no es un diari de modes $[\ldots]$ Y, com, es la nostra missió l'enaltiment de la dòna en totes les esferes, y la seva cultura, y hem fet de nostres planes un camp obert a totes les iniciatives qui de prop 
La presentación material de la revista tenía gran importancia, el papel de tipo couché tenía un gran formato y se utilizaban dos tintas para mejorar la calidad de las imágenes, de relevancia para la editorial. Sus páginas iban ilustradas con dibujos, grabados y pinturas, la mayoría de ellos realizados por la pintora Llüisa Vidal; pero sobre todo con fotografías, invento relativamente reciente y que no todos los medios podían permitirse debido a su elevado coste.

Podemos distinguir diversas secciones en la revista Feminal, entre ellas: artículos de fondo configurados por la opinión sobre asuntos feministas o sobre otros temas (escritos por la propia directora o colaboradores), situación de la mujer en diferentes lugares de la geografía española, crítica de libros, reportajes o crónicas extranjeras, interiores de casas de la alta burguesía, acontecimientos sociales, labores, moda y reportajes de la vida y obra de mujeres relevantes. Además, también podemos encontrar una página literaria que incluye una narración corta o poema, una página musical con una composición musical y, por último, publicidad con anuncios sobre productos de belleza, ropa, medicina, higiene, etc.

\section{El pensamiento ideológico en la Revista FeminaL}

El pensamiento ideológico que Carme Karr plasmó en Feminal está estructurado en torno a tres ejes: la educación, la profesionalización de la mujer y la reivindicación del derecho al voto.

En relación al primero de ellos, la educación, Carme Karr enuncia que el objetivo de la revista es la cultura de la mujer'. La autora no entra en la discusión de si las mujeres son menos inteligentes que los hombres, según sus propias palabras: «A la mayoría de las mujeres catalanas de hoy

ò de lluny poden servirli. Que vinguin donchs a nosaltres totes les dones: les escriptores, poetises, pedagogues; les pintores, dibuixantes, les músiques, les artistas, les sociòlogues, les pacifistes, les estudiantes. Que vinguin també les artesanes; quese'ns acullin sense cap temensa totes aquelles qui aspiren a esser algú. Ò a produhir quelcom en la obra social, artística, literaria, industrial, científica, etc., y a portarhi el seu petit grà de sorra».

9 Ref. Carme Karr: Op. Cit., 1, p. 2v. (Ana Muñoz, trad.). A continuación, se transcribe la cita original: «revista destinada especialment a la cultura de la dòna». 
no les falta inteligencia, pero son en cambio de una gran ignorancia y esta ignorancia las esclaviza y las hace $(. .$.$) envidiosas» { }^{10}$.

En los artículos denominados Consideraciones ${ }^{11}$, Carme Karr entra de lleno en el tema de la ignorancia, analizando las causas que la provocan y ofreciendo alternativas. Este punto ya había sido tratado por algunas de sus predecesoras, como Dolors Monserdà, que tenían una postura contraria hacia las teorías científicas que atribuían a las mujeres una menor capacidad intelectual que la de los hombres ${ }^{12}$. Según la directora de Feminal, algunas de las causas que provocan esta ignorancia son las enseñanzas en los internados religiosos, donde no existe higiene, no se realiza ejercicio físico, los profesores tienen poca preparación y las niñas terminan sus estudios con 15 años ${ }^{13}$. Después de los 15 años, estas niñas continúan su preparación con institutrices poco preparadas, hasta que se casan. Otro de los motivos de la deficiente educación femenina es debido a los padres, ni el padre practica su paternidad ni la madre su maternidad ${ }^{14}$. Según Karr, «los padres son los culpables de este estado moral e intelectual de sus hijas, por esta confianza refinada, esta indiferencia con que han dejado que se eduquen en nuestras instituciones, $\tan$ deficientes ${ }^{15}$.

Se ofrecen en la revista diversos modelos educativos que se están impartiendo en Europa, Inglaterra y Estados Unidos, creándose además diversas instituciones que surgen desde esta publicación, como la residencia para estudiantes La Llar, La Biblioteca Popular per a la dòna, el Centre de Cultura femenina, el Centro de cultura popular o el Institut Feminal, entre otros.

10 Ref. Carme Karr: Op. Cit., 68, p. 1v. (Ana Muñoz, trad.). A continuación, se transcribe la cita original: «La generalitat de les dones catalanes d'avuy no es mancada d'inteligencia, però es en camvi d'una palesa i desoladora ignorància i aquesta ignorancia l'esclavisa i la fa (...) envejosa».

11 Se encuentra en los artículos titulados Consideracions. Cfr. en Carme Karr: Op. Cit., 1, p. 2v; 33, p. 1v; 34, p. 1v; 35, p. 1v; 36, p. 1v; 37, p. 1v y 38, p. 1v.

12 Ref. Monserdà de Macià, Dolors: Estudi feminista $i$ orientacions per a la dòna catalana (Barcelona: Lluis Gili, 1909).

13 Ref. Carme Karr: Op. Cit., 30, pp. 1v-2r.

14 Ref. Carme Karr: Op. Cit., 31, p. 1v.

15 Ref. Carme Karr: Cultura femenina: Estudi i orientacions (Barcelona: Tip L'Avenç, 1910), p. 77. (Ana Muñoz, trad.). A continuación, se transcribe la cita original: «Els pares són els culpables d'aquest estat moral y intelectual de les llur filles, per aquesta confiança refinada, aquesta indiferencia amb que han deixat que s'eduquessin en les nostres institucions, tant deficients». 
Los argumentos que se dan a lo largo de la revista para que la mujer se eduque son dos:

$\left.1^{\circ}\right)$ El matrimonio: los hombres de sociedad no se casan debido a la poca instrucción de las mujeres ya que ellas no les comprenden y, por tanto, ellos no las ven como la compañía ideal. Según Carme Karr:

\section{Las chicas llegan al mercado matrimonial siendo muy poco competitivas, o} tenian una dote o no podian ofrecer nada más. Pero no todas las mujeres tenian dotes por entregar, por tanto, si queremos recuperar los indices matrimoniales y las tasas de natalidad que están bajando de manera alarmante en estosprimeros años del nuevo siglo, habrá que dotar a las mujeres de un nuevo utensilio: la cultura; cultura e instrucción profesional y educación casera. ${ }^{16}$

$2^{\circ}$ ) La instrucción de los niños pequeños, ya que la madre es la encargada de impartir estas primeras enseñanzas, por lo que para ello debe estar preparada.

Sobre el segundo de los aspectos, la inserción laboral, Carme Karr reivindica el acceso a todas las profesiones, rompiendo con los prejuicios que condenaba al sector femenino a reducidos ámbitos de actuación. Por otra parte, denuncia las deplorables condiciones laborales en que vivían inmersas amplios sectores de mujeres, como la explotación económica de la asalariada. Según ella, el empresario paga menos si se considera femenina la profesión y escribe «las mujeres aceptan los empleos porque les urge trabajar y encuentran faena, naturalmente, porque una cajera sin estudios y que cumple con su trabajo, gana un sueldo de 12 a 15 duros mientras que un hombre cobraría de 25 a 30 duros» ${ }^{17}$.

16 Ref. Carme Karr: Op. Cit., 34, pp. 1v-2v. (Ana Muñoz, trad.). A continuación, se transcribe la cita original: «Les noyes arriben al mercat matrimonial, essent molt poc competitives, o tenen una dot o no poden oferir res més. Però no totes les dones tenen dots per a entregar, per tant, si volem recuperar els índex matrimonials i les taxes de natalitat que están baxant de manera alarmant en aquests primers anys del nou segle, caldrà dotar a la dona de un nou estriu: la cultura; cultura $i$ instrucció profesional i educación casolana».

17 Ref. Carme Karr: Op. Cit., 63, pp. 1v-2v. (Ana Muñoz, trad.). A continuación, se transcribe la cita original: «Les dones d'altra banda accepten els empleus car els urgeix treballar i troben feïna, naturalment, perquè una caixera sense estudis i que compleix amb el seu treball, guanya un sou de 12 a 15 duros mentres qu'un home cobraria de 25 a 30 duros $[\ldots] \gg$. 
Se crean diversas asociaciones para evitarlo como Acció Social de la dòna, los Patronatos escolares de obreras y la Federación sindical de obreras, entre otras. Además, para Karr algo también denunciable es el mercado de prostitución que esconden algunas de las ofertas laborables, cuestiones de las que también se encargan estas instituciones.

Una cuestión interesante, a lo largo de la revista, es el comentario de la situación femenina en otros países. Se citan a mujeres que se han profesionalizado, de las cuales se realiza un comentario en la mayoría de números de la revista, cosa que ha convertido a Feminalen una extraordinaria fuente de documentación sobre la vida y obra de muchas de las profesionales de las primeras décadas del siglo XX.

Sobre el último aspecto ideológico que se estudia en la revista, la reivindicación del derecho al voto, puede observarse como Carme Karr en prácticamente toda la revista centra su interés en aumentar la cultura de la mujer pero no en pedir el voto femenino al opinar que antes de que la mujer pudiera votar necesitaba aumentar su cultura ya que, en caso contrario, su voto no era útil. Sin embargo, en el último año de publicación de la revista, puede apreciarse como cambia su opinión sobre este tema y denuncia que la mujer no pueda votar o ser elegida para defender sus intereses, es posible que sea debido por el escaso interés que los dirigentes prestaban a las reivindicaciones feministas de la autora:

¡El voto de la mujer!

¡Por qué caminos de sangre, de duelo y de sacrificios habremos de conseguir este derecho que se nos debia desde la primera legislación que se hizo en la tierra! (...)

$\dot{¿}^{N o}$ es la mujer contribuyente del Estado en iguales condiciones que el hombre? ¿Existe para ella rebaja especial (por ser mujer y no tener derecho al voto) en las tarifas de cualquier clase? (...)

¿Por qué no podría, pues, ser elegida la mujer para defender en las Corporaciones municipales, provinciales, en el Congreso, en las Cortes, aquellos intereses femeninos que forman una parte integrante de la nación? (...) Ya he citado Suecia y Noruega; podría hacerlo también con Finlandia y Dinamarca, asi como con algunos Estados de EEUU, en que el voto de la mujer es más que beneficioso. (...) 
Va llegando la hora de la justicia en nuestros derechos, y el futuro demostrará a los hombres que necesitan a la mujer para conseguir aquella forma de gobierno ideal soñada por Platón en su República, es decir: el gobierno de los buenos por los mejores ${ }^{18}$.

\section{El Repertorio musical pUblicado EN FEMinaL}

Consideramos imprescindible el comentario de las obras musicales, aparecidas en la revista Feminal, como un punto independiente debido a la relevancia que poseen dentro de la publicación y como corpus musical dentro de la cultura catalana. El repertorio musical de Feminal es peculiar por varios motivos. El primero de ellos es el medio donde se han publicado, aunque era habitual la existencia de suplementos musicales con partituras destinadas al sector femenino ${ }^{19}$ resulta más extraño que estas partituras aparezcan por primera vez, en la ciudad de Barcelona, publicadas en una revista feminista escrita por y para mujeres, cuyo objetivo primordial era el aumento de la cultura femenina ${ }^{20}$. El segundo motivo es el elevado porcentaje de mujeres $(78 \%)$ en relación al de hombres (22\%) que componen las piezas musicales publicadas en Feminal, en un momento sociocultural donde era extraño que las mujeres compusieran música, ya que en estos años no se tenía buena concepción de aquellas mujeres que se dedicaban a la composición.

18 Ref. Carme Karr: Op. Cit., 124, pp. 1v-3r. El texto transcrito es el arreglado en la publicación de Josep M. Casasus: Periodisme català que ha fet bistoria (Barcelona: Proa, 1996), pp. 168-169. (Ana Muñoz, trad.). A continuación, se transcribe la cita original: (...) ¡El vot de la dona!/ ¡Per quins camins de sang, de dol i de sacrificis haurem conseguit aquest dret que se'ns devia des de la primera llegislació que va fer-se damunt la terra! (...)/ ¿No és la dona contribuient de l'Estat en iguals condicions que l'home? ¿Existeix per ella rebaixa especial (per ésser dona i no tenir dret al vot) en les tarifes i aranzels de qualsevol classe? (...) / ¿Per què no podria, doncs, ésser elegida la dona per defensar en les Corporacions municipals, provincials, al Congrés, a les Corts, aquells interessos femenins que són una part integrant de la nació? (...)/ Ja he citat Suècia i Noruega; podria fer-ho també amb Finlàndia i Dinamarca, aixís com alguns Estats de la Unió Americana, en què el vot de la dona és sols beneficiós. (...) / Va arribant l'hora de la justícia als nostres drets, i el pervindre demostrarà als homes que necessiten a la dona per conseguir aquella forma de govern ideal somniada per Plató en sa República, això es: el govern dels bons pels millors».

19 «La cultura de adorno» de cualquier mujer de la burguesía o aristocracia incluía lecciones de canto y piano. Cfr. en Matilde Olarte Martínez. «Situación de los estudios musicales para la mujer española a comienzos de nuestro siglo», Enrique Banus (Ed.). El espacio social femenino (Pamplona: Newbook Ediciones, 2000), pp. 531-532.

20 Isabel Segura y Marta Selva realizan un estudio de las revistas de mujeres publicadas en Barcelona desde 1846 hasta 1935. Cfr. en Isabel Segura y Marta Selva. Revista de dones (18461935) (Edhasa: Barcelona, 1984). 
Concretamente, en 1914, Joaquín Turina escribe las siguientes líneas en la Revista Musical Hispanoamericana:

La mujer puede abordar el estudio de los instrumentos, y en ocasiones puede hasta aventajar al hombre en finura y refinamiento; mas niego en absoluto que la mujer tenga condiciones para la composición. Afortunadamente, en España se desconoce aún esta terrible plaga de compositoras y eruditas; en París, iqué horror, Dios mío!...estudian la composición con mucho más abinco que el hombre, y con la facilidad de memoria de que son capaces (...) deciden ser eruditas y hacer criticas (...)

Pero estas eruditas, ¿son realmente mujeres? Yo estoy por dudarlo, pues á fuerza de estudios empiezan á secarse, y ya pálidas y macilentas, con los sempiternos lentes y los enormes cartapacios llenos de partituras con anotaciones, más parecen escribanos que representantes del bello sexo. (...) si el cultivo de un instrumento perfecciona á la mujer, el enorme esfuerzo que representa asimilarse el estudio de la composición, las deprime y seca, y á la verdad, yo te aseguro que para secos y feos nos bastamos á nosotros mismos ${ }^{21}$.

Todas las peculiaridades comentadas del repertorio musical se responden al analizar la revista ya que Carme Karr, su directora, pretende a lo largo de toda la publicación aumentar la participación de las mujeres en cualquiera que sea el campo de acción, como el educativo, el cultural, el laboral o el del derecho al voto, es decir, pretende acercar los derechos de las mujeres a los derechos que ya poseían los hombres; y en el terreno musical ocurre exactamente lo mismo, al formar éste parte de la educación del sector femenino de las clases elevadas y como parte de la cultura de la región catalana.

El repertorio musical de la revista pertenece al género de salón. La música de salón tenía una misión de entretenimiento, pero también era el ornamento indispensable para una reunión social. Por estas razones, el género de salón dependía de los imperativos de la moda y, poco a poco, aumentaba su público. En el último tercio del siglo XIX, los salones españoles acogen un repertorio musical que se puede resumir en cuatro grandes grupos ${ }^{22}$ :

21 Ref. Joaquín Turina. «El feminismo y la música», Revista musical hispano-americana, 2 (febrero 1914), pp. 8-9.

22 Ref. Celsa Alonso: Op. Cit., p. 455. 
1.-Piezas procedentes de óperas italianas y francesas y algunas romanzas de zarzuela.

2.- Obras para piano a dos y cuatro manos.

3.- Obras vocales en castellano: canciones españolas y andaluzas y en la segunda mitad del siglo XIX algunas romanzas y habaneras.

4.- Escasas representaciones de alguna obra dramática con o sin música.

Sin embargo la situación en Cataluña era diferente a la del resto de España, frente a la madrileña, la burguesía catalana tuvo un gran ascenso en el último tercio del siglo XIX, en parte debido a su apertura al mercado americano. Fueron años de cambios importantes, desde la restauración de la lengua catalana, a la importancia por la reivindicación de la cultura propia y, más tarde, al florecimiento de la poesía en catalán, apareciendo poetas importantes como Víctor Balaguer, Apel·les Mestres, Joan Maragall o Jacinto Verdaguer.

En este proceso la cançó catalana y los lieder catalanes tuvieron un peso muy relevante cimentados en una rica poesía en lengua nativa e, inicialmente, bajo un aliento romántico: no en vano la influencia de la cultura alemana había sido más profunda en Cataluña que en el resto del país desde mediados del siglo XIX. En este sentido, la cançó catalana ${ }^{23}$ suponía la consagración de un elemento vivo que respondía a las más íntimas esencias comunitarias, razón por la cual habría de ser el factor generador de un arte lírico-musical genuinamente catalán.

Al mismo tiempo tuvo lugar un progresivo afianzamiento de la música de cámara en los salones de la burguesía y el fortalecimiento del wagnerismo, culminando con la creación de la Associació Wagneriana en 1901 por Joaquim Pena. A la creación de la Societat de Concerts hay que añadir la fundación de la Sala Beethoven, «uno de los pilares de la renovación musical no operística» durante los últimos veinte años del siglo XIX, que marca el inicio del ascenso de la música de cámara en la sociedad barcelonesa, fundándose en 1913 la Associació de Música da Camera liderada por Jacint Raventós. En 1881, se creó la revista L'Avenç que

23 Denominación utilizada por Celsa Alonso, Cfr. Celsa Alonso: Op. Cit. p. 481. 
apadrinó a la regeneración de la cultura catalana en clave modernista, unida a la asimilación del wagnerianismo y de la escuela franco-belga. En esta revista colaboró Carme Karr, por lo conocía la orientación que se estaba llevando a cabo en la música catalana.

El progresismo político se tradujo en una orientación europeísta del arte catalán, los compositores catalanes tomaron conciencia de la trascendencia del lied en el proceso de identificación nacional, sobre el fermento del conocimiento del romanticismo alemán. Tras la aportación crucial de Pedrell ${ }^{24}$, folklorismo, nacionalismo, neorromanticismo, europeísmo y modernismo presiden la creación del lied catalán: se asumieron los modelos franceses y alemanes sobre un sustrato de asimilación de la cançó catalana. Por lo que, tras los lieder y cançons catalanas de autores como Juan Goula, José Rodoreda, Roberto Goberna, Francisco Sánchez Gabañach, Candido Candi Candi, Francisco Fors de Casamayor, Françesc Alió y Fermín Alvarez, se despliega la obra coral de Antonio Nicolau y lírica de Joan Borrás de Palau, Narcisa Freixas, Apel·les Mestres, Enric Morera, Julio Garreta o Joan Lamote de Grignon, entre otros, bautizados por Pedrell como los «preludios catalanes de la música española del porvenir» ${ }^{25}$.

En este repertorio musical podemos encontrar plasmado parte de este pensamiento musical: son piezas tonales con un sustrato romántico para vOz y piano que utilizan poemas de autores catalanes, predominando los autores Apel·les Mestres y Jacinto Verdaguer. Algunos de los compositores son los bautizados por Pedrell como los «preludios catalanes», es decir, Narcisa Freixas, Juli Garreta y Joan Borrás de Palau, aunque también algunos de los compositores de lieder catalanes o de música nacionalista fueron profesores

24 Pedrell pretendía concebir un lenguaje libre de italianismos y casticismos. Para lograrlo era preciso profundizar en las riquezas del canto popular y prestar atención a los logros de los compositores franceses en el dominio de la melodie. El análisis del canto popular se revelaba como una primera tarea a realizar, no en vano para Pedrell el drama lírico era el «Lied engrandecido», es decir, el «canto popular transformado», donde había de residir «el temperamento artístico de un pueblo del que emana, por consiguiente, su carácter. Por lo tanto, tres parecen ser los elementos que para Pedrell configuran el perfil estético de la canción lírica: sabia popular, romanticismo y europeísmo. Ideas extraídas del manual de Celsa Alonso: Op. Cit., pp. 435-438.

25 Las ideas extraídas sobre la cançó catalana se encuentran en Celsa Alonso: Op. Cit., pp. 480-482. 
de algunas de las compositoras como Francisco Sánchez Gabañach y Felipe Pedrell o Granados y Falla ${ }^{26}$.

Aunque todas las piezas son tonales, en algunas de ellas de ellas se produce un debilitamiento de los fundamentos estructurales de la tonalidad tradicional, con la finalidad de una búsqueda de una determinada modernidad compositiva. Se puede apreciar la utilización de elementos de la literatura y estética modernista catalana y el empleo de elementos medievales como medio de revalorización de lo vernáculo; ambas como características del ideario de Pedrell sobre la cançó catalana.

\section{Conclusiones}

A través del presente artículo se ha pretendido acercar al lector la revista Feminal; una de las publicaciones que ha formado parte de la historia del feminismo español. Se conoce, gracias al estudio de Isabel Segura y Marta Selva ${ }^{27}$, que en la ciudad de Barcelona Feminal fue la primera revista que reivindicaba el acercamiento de los derechos de la mujer a los que ya poseían los hombres pero sería necesario ampliar este estudio a la región catalana y al resto del país para poder completar la historia del feminismo español con la información contenida en las publicaciones.

La historia del feminismo de nuestro país no es sólo una historia de publicaciones sino también de grandes personalidades que han ayudado a que el género femenino tenga actualmente derechos que a principios del siglo XX no poseía y, aunque todavía, el proceso no ha llegado a su meta final, consideramos necesario el comprender lo realizado hasta el presente para poder continuar en el avance de la igualdad de los derechos de todos.

26 Francisco de Paula Sánchez Gabagnach dio clase a María Davalillo, Lluïsa Casagemas Coll y María Carratalà; Felipe Pedrell fue profesor de esta última compositora. Margarida Orfila recibió clases de Millet, Lamote de Grignon y Granados. Y, por último, Rosa Garcia Ascot tuvo como profesores a Enrique Granados y Manuel de Falla.

27 Ref. Isabel Segura y Marta Selva: Revista de dones (1846-1935) (Edhasa: Barcelona, 1984). 


\section{Bibliografía}

Adkins Chiti, Patricia: Las mujeres en la música (Madrid: Alianza Editorial, 1995)

Ainaud de Lasarte, Josep María: "Carme Karr, escriptora i feminista", Serra d'Or, 409 (1984), pp. 20-23

---: Carme Karr (Barcelona: Infiesta, 2010)

Almirall, Valentí: Lo catalanisme. Motius que'l llegitiman. Fonaments científichs y solucions prácticas (Barcelona: Alta Fulla, 1978)

Alonso, Celsa: «Los salones: un espacio musical para la España del XIX», Anuario musical: Revista de musicología del CSIC, 48

Alonso, Celsa: La canción lírica española en el siglo XIX (Madrid: ICCMU, 1988)

Casasus, Josep M: Periodisme català que ha fet historia (Barcelona: Proa, 1996)

Karr, Carme: Cultura femenina: Estudi i orientacions (Barcelona: Tip L'Avenç, 1910)

Karr, Carme: Feminal (Barcelona: Establiment gràfich Thomas, 19071917)

Monserdà de Macià, Dolors: Estudi feminista i orientacions per a la dòna catalana (Barcelona: Lluis Gili, 1909)

Olarte Martínez, Matilde. «Situación de los estudios musicales para la mujer española a comienzos de nuestro siglo», Enrique Banus (Ed.). El espacio social femenino (Pamplona: Newbook Ediciones, 2000)

Roselló, Isabel: Música, femení singular. Dones compositores, una ullada a la bistoria (Barcelona: Di7, 1998)

Segura, Isabel; Selva, Marta: Revista de dones (1846-1935) (Edhasa: Barcelona, 1984)

Segura, Isabel: Guía de dones de Barcelona ([Barcelona]: Ajuntament de Barcelona, 1995) 
Segura, Isabel: Els feminismes de "Feminal" (Barcelona: Institut Català de les Dones, 2007)

Turina, Joaquín: «El feminismo y la música», Revista musical bispanoamericana, 2 (febrero 1914) 